Sharif University of Technology
Scientia Iranica
SCIENTIA
I RAN I CA
http://scientiairanica.sharif.edu

\title{
Experimental research on adfreezing strengths at the interface between frozen fine sand and structures
}

\author{
S. Quanbin, Y. Ping*, and W. Guoliang \\ Institute of Civil Engineering, Nanjing Forestry University, Nanjing, Jiangsu, China. \\ Received 23 May 2016; received in revised form 26 November 2016; accepted 28 January 2017
}

\author{
KEYWORDS \\ Frozen fine sand; \\ Residual adfreezing \\ strength; \\ Sand-structure \\ interface; \\ Peak adfreezing \\ strength; \\ Variation law.
}

\begin{abstract}
To explore the mechanisms concerning adfreezing strengths at the interface between frozen fine sand and structures, a series of experiments were conducted using a direct shear apparatus. The main results are as follows: All adfreezing strengths increased with decreasing temperature and increasing normal stress and surface roughness. Peak adfreezing strength (peak shear stress at failure) had a linear relationship with temperature, its relationship with normal stress was in line with the Mohr-Coulomb criterion, and its relationship with roughness satisfied a logarithmic function. Residual adfreezing strength (post-peak shear stress) varied with temperature and normal stress, and three typical regular patterns were presented: sustained stable, first stable and then fluctuating, and circularly fluctuating. The fluctuation cycle about strength curves increased with increasing roughness. An empirical formula on adfreezing strengths incorporating temperatures, normal stress, and roughness was constructed.
\end{abstract}

(C) 2018 Sharif University of Technology. All rights reserved.

\section{Introduction}

The shield method is commonly used in subway construction, where the start and end of the tunnel as well as the contact channel are the key risk control points. In soft soils, soil reinforcement in the contact channel and the ends of the tunnel is, therefore, a decisive factor in tunnel construction safety. A variety of engineering methods are used to reinforce soil; for a soft, water-rich sand environment, artificial freezing is commonly used. However, adfreezing force occurs at the interface between frozen soil and structure in this method, and the structure may include shield segment,

\footnotetext{
*. Corresponding author. Tel./Fax: +86 2585427773 E-mail addresses: 13338888183@163.com (S. Quanbin); yangping@njfu.edu.cn (Y. Ping);271475341@qq.com (W. Guoliang).
}

doi: $10.24200 /$ sci. 2017.20005 shield machine shell in artificial frozen soil area, pile foundation in permafrost regions, etc. This adfreezing force causes redistribution of the structural stress field, or even causes damage to tunnel segments. When a horizontal freezing technique is used at the shield originating or receiving stage, the shield housing and cutter may freeze due to adfreezing if the frozen soil temperature is too low, making the proper advancement of the lead shield more difficult. Therefore, the research on adfreezing force and adfreezing strength at the interface between artificially frozen soil and structural elements has important practical significance in tunnel engineering and may lead to more reliable tunnel construction using the shield method.

Several researchers [1-9] did some experimental and numerical studies on the general behavior of geomaterial interfaces. However, at present, research results on adfreezing strength at the interface between artificially frozen soil and structures are relatively few and basic. Concerning adfreezing strengths, 
Parameswaran [10], Ladanyi and Theriault et al. [11], Terashima et al. [12], and Weaver and Morgenstern [13] studied adfreezing strengths between frozen soil and piles. Jianming and Zhang [14] pointed out that the decrease speed of adfreezing strength under dynamic loading was higher than that under static loading. The adfreezing strength of model piles reached its maximum when the moisture content of frozen soil approached the saturation value. The rigidity of concrete piles was a more obvious influence on adfreezing strength than that of steel-pipe piles.

In view of high-speed railway construction in western China, a new requirement for the study of adfreezing strength under dynamic loads was proposed. Liu et al. [15] and Lv et al. [16] developed a frozen soil direct shear instrument for dynamic shear strength testing and made general conclusions where the dynamic shear strength at the interface between frozen soil and concrete was affected by temperature, normal stress, water content, roughness, loading cycles, etc. However, they did not provide a specific explanation of the adfreezing strength or its variation laws.

On adfreezing strengths of different soils, Biggar and Sego [17] researched adfreezing strength between frozen saline soil and bored pile. They found that temperature and salinity influenced the content of unfrozen water, and then had an impact on adfreezing strength. Ueda et al. [18] studied the single effective factor of normal stress, and pointed out that adfreezing strength of frozen sand and frozen clay interfaces all increased with increasing normal stress; in addition, the increase rate of frozen sand was faster than that of frozen clay.

In terms of adfreezing strength calculation, Choi and Ko [19], Lee et al. [20], Ko and Choi [21], Lee and Choi [22] and Lee and Choi [23] proposed a new method that used a conversion coefficient to calculate adfreezing strength between frozen soil and structures based on the shear strength of frozen soil. Liu et al. [24] carried out direct shear tests, and discovered that shear strength had linear relations with normal pressure and temperature, but a nonlinear relation with water content. However, the influence of the roughness of the shear plate was not considered in these direct shear tests; therefore, significant variations in residual adfreezing strength were not found. They had no thorough explanation of these phenomena from the standpoint of the formation mechanism of adfreezing strength. Although they presented fitted equations between factors and shear strengths, the equations could not incorporate all the various factors that affected adfreezing strength.

Zhao et al. [25], who are members of the present research group, developed a large-scale, multifunctional, frozen soil circulation direct shear apparatus, which provided good conditions for studies of the frozen soil-structure interface by Zhao et al. [26-27] and Sun et al. [28].

In summary, the aforementioned studies on adfreezing strength were carried out as experimental studies or numerical analyses. These studies came to a preliminary understanding concerning the effects of temperature or normal stress and other single factors on adfreezing strength. However, there has not been a thorough explanation on the inherent formation mechanism of adfreezing strength, or a detailed classification of adfreezing strength. No laws have been formulated on variations of residual adfreezing strength, nor have there been multivariate quantitative analyses of the multiple factors influencing adfreezing strength. Therefore, in this paper, a series of direct shear tests at the interface between frozen soil and structures were carried out by means of a self-developed, large-scale, frozen soil direct shear apparatus.

\section{Test equipment and test programs}

\subsection{Test equipment}

To carry out this project, a self-developed, largescale, frozen soil, direct shear apparatus (DDJ-1 type) was used whose main components included a loading system (including horizontal and vertical loading devices), a sensor system (including horizontal and vertical displacement sensors, horizontal and vertical load sensors, and a temperature sensor), a refrigeration and temperature control system, a data acquisition and self-stabilizing system, and an auxiliary unit. For the apparatus's specific configuration and testing parameters, see [25].

\subsection{Test scheme}

\subsubsection{Test material}

Nanjing city is located in the Yangtze River floodplain area, where fine sand is widely distributed and a number of subway lines pass through the fine sand layer. For tunnel construction in these regions, strengthening soil by artificial freezing is often used adjacent to the shield originating and receiving ends and the contact channel. So, the typical fine sand of Nanjing city was chosen for this study. The main properties of the sand are shown in Table 1, and its particle size distribution curve is shown in Figure 1. In this experiment, samples of fine sand were remolded in accordance with undisturbed fine sand (its water content was $26 \%$ ).

The soil sample was remolded in conformity with the original soil water content and layered into the copper soil sample box, whose inside shape was a rectangular block (length $200 \mathrm{~mm}$, width $100 \mathrm{~mm}$, height $87 \mathrm{~mm}$ ). The soil sample was remolded into the soil sample box in three layers. Each layer was compacted evenly and densely, such that the average density of the soil sample was $1.96 \mathrm{~g} / \mathrm{cm}^{3}$. Finally, the 
Table 1. Physical and mechanical properties of the Nanjing silty fine sand.

\begin{tabular}{|c|c|c|c|c|c|}
\hline $\begin{array}{c}\text { Water } \\
\text { content, } \\
\omega(\%)\end{array}$ & $\begin{array}{c}\text { Bulk } \\
\text { density, } \\
\gamma\left(\mathrm{kN} / \mathrm{m}^{\mathbf{3}}\right)\end{array}$ & $\begin{array}{l}\text { Void } \\
\text { ratio, } \\
e\end{array}$ & $\begin{array}{l}\text { Coefficient of } \\
\text { compressibility, } \\
\quad a\left(\mathrm{MPa}^{-1}\right)\end{array}$ & $\begin{array}{c}\text { Modulus of } \\
\text { compression, } \\
E_{s}(\mathrm{MPa})\end{array}$ & $\begin{array}{c}\text { Angle of } \\
\text { internal friction, } \\
\phi(\mathrm{deg})\end{array}$ \\
\hline 26.0 & 19.8 & 0.721 & 0.145 & 12.98 & 30.2 \\
\hline
\end{tabular}

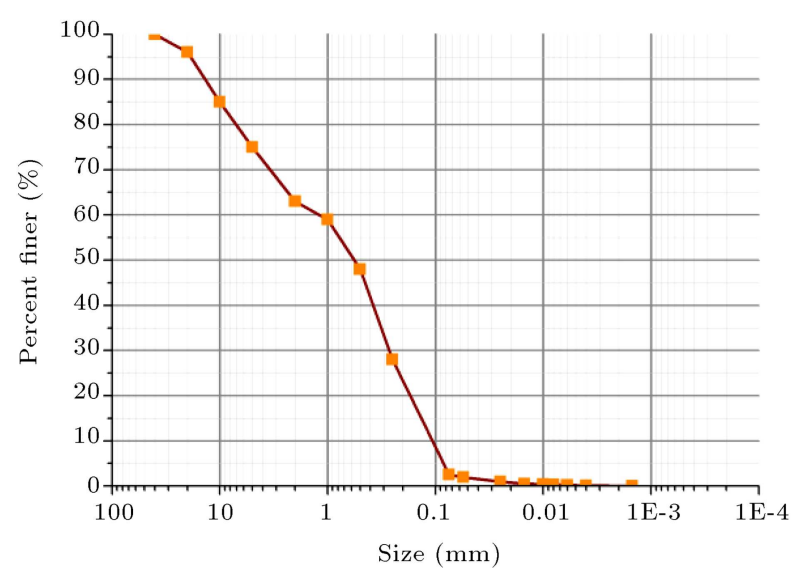

Figure 1. Particle size distribution curve of Nanjing silty fine sand.

soil sample surface was trimmed smoothly with a soil repairing knife.

\subsubsection{Test programs}

To account for the factors most closely related to adfreezing strength of the interface between frozen sand and structures, this project used four factor tests and three level tests: normal stress at the interface, $\sigma$ (100 kPa, $300 \mathrm{kPa}, 500 \mathrm{kPa}$, and $700 \mathrm{kPa}$ ), shear plate roughness, $R(0 \mathrm{~mm}, 0.3 \mathrm{~mm}, 0.8 \mathrm{~mm}$ and $1.4 \mathrm{~mm})$, and interface temperature, $T\left(-2^{\circ} \mathrm{C},-6^{\circ} \mathrm{C}\right.$, $-10^{\circ} \mathrm{C}$, and $-14^{\circ} \mathrm{C}$ ), a total of 64 tests. Because shear plate replacement took a long time, it greatly influenced the test progress; therefore, the tests were divided into different groups according to the roughness value of the shear plate. Each group was subjected to different interface temperatures and normal stress and underwent a total of 16 combination tests. The test parameters of the direct shear apparatus were as follows: shearing amplitude $11 \mathrm{~mm}$, shearing rate 5 $\mathrm{mm} / \mathrm{min}$, data acquisition cycle 1 time $/ 2.5 \mathrm{sec}$.

The direct shear apparatus used in this project had been improved so that the surface area of the shear plate could be larger than that of the contact area between the soil and the plate, and thence the contact surface could be kept constant during the direct shear process. Therefore, shear stress and normal stress did not have to be modified for reducing area.

\subsubsection{Refrigeration and temperature control system}

The DDJ-1 frozen direct shear apparatus for refrigeration and temperature control systems comprised a copper soil sample box, a low-temperature (isothermal)

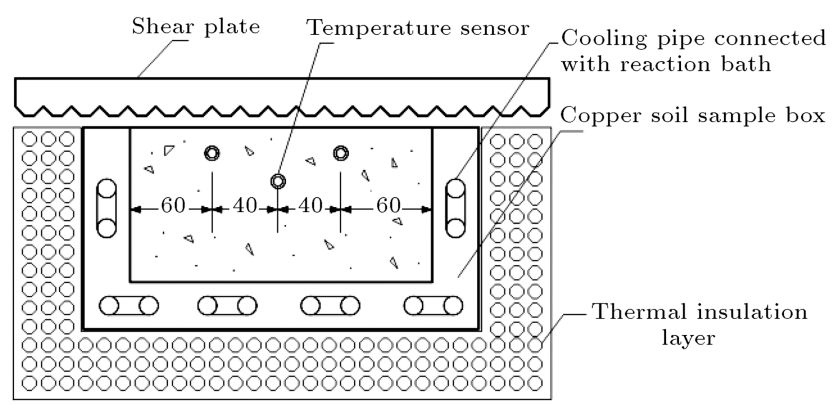

Figure 2. Schematic diagram of refrigeration and temperature control system $(\mathrm{mm})$.

mixing reaction bath (power $2 \mathrm{kw}$ ), and temperature sensors, as shown in Figure 2. The lowest control temperature of the mixing reaction bath was $-25^{\circ} \mathrm{C}$, the temperature control range of the stability test was $0^{\circ} \mathrm{C}$ to $-20^{\circ} \mathrm{C}$, and the temperature control precision was $\pm 0.3^{\circ} \mathrm{C}$. To measure temperature, six PT-100 platinum thermal resistance temperature sensors were used; their measurement accuracy was $\pm 0.1^{\circ} \mathrm{C}$. All temperature sensors were arranged in the soil sample box in two layers: four in the upper set and two in the lower set. The measured average values of six temperature sensors were taken as the measurements of temperature. According to the temperature measurements, the temperature control system adjusted the reaction bath to achieve thermal self-stabilization.

\section{Definition and classification of adfreezing strengths}

In study of Qiu et al. [29], the definitions of adfreezing force and adfreezing strengths are as follows: adfreezing force is the shear force by which the interface between the side surface of a foundation and frozen soil could transmit; adfreezing strength is the maximum shear stress that the interface between the side surface of a foundation and frozen soil could bear.

Based on the relevant literature and the research results of this project, we divided adfreezing strength at the interface between frozen sand and structures into two types: peak adfreezing strength and residual adfreezing strength. The moment when the shear stress reaches its maximum and begins to slip is called the critical state of shear slip damage. Peak adfreezing strength is the peak shear stress at the critical state of shear slip damage, commonly called adfreezing strength. Residual adfreezing strength is the post- 


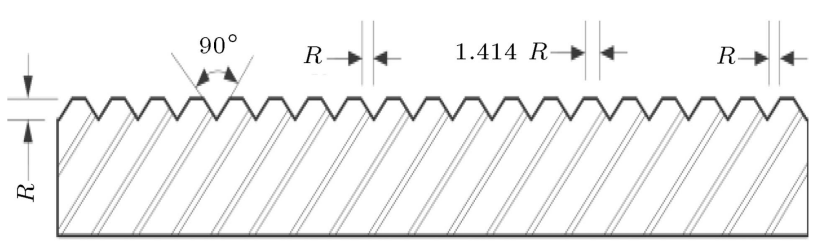

Figure 3. Longitudinal profiles of rough shear plate.

peak shear stress after the shear slip damage, and its value varied with the relative shear displacement. Peak adfreezing strength is more meaningful for engineering practice; therefore, this article focuses on quantitative analysis of the peak adfreezing strength and only qualitative analysis of residual adfreezing strength.

\section{Analysis of factors influencing adfreezing strengths}

The relation curves between shear stress, $\tau$, and shear displacement, $\mu$, could clearly reflect variations in adfreezing strength, so factors influencing adfreezing strength were analyzed based on $\tau$ versus $\mu$ curves.

A shear steel plate with grooves of different depths was used to simulate different structural roughnesses; Figure 3 is a longitudinal profile of the plate. Interface roughness value, $R$, was defined as the height of the trapezoidal teeth of the plate groove, measured in millimeters. Shield segment surface is smooth, and its surface roughness value is generally $0.2 \mathrm{~mm}$; concrete pile surface is relatively rough, and its surface roughness value is generally $1.2 \mathrm{~mm}$. In order to study the influence of roughness on adfreezing strength of contact surface, considering the surface roughness of two kinds of typical underground structure above, the surface roughness values were chosen to be $0.0 \mathrm{~mm}$, $0.3 \mathrm{~mm}, 0.8 \mathrm{~mm}$, and $1.4 \mathrm{~mm}$.

\subsection{The influence of temperature on adfreezing strengths}

In tunnel construction using artificial freezing, the average temperature of the freezing wall is generally $-10^{\circ} \mathrm{C}$, and the interface temperature between frozen soil and structure is usually not uniform. To study the influence of temperature on the adfreezing strength of the interface, we tested the strengths at four temperatures: $-2^{\circ} \mathrm{C},-6^{\circ} \mathrm{C},-10^{\circ} \mathrm{C}$, and $-14^{\circ} \mathrm{C}$.

Due to similar laws of $\tau$ versus $\mu$ curves under different stresses, for instance, we only show these $\tau$ versus $\mu$ curves for each temperature under constant roughness $(R=0.0 \mathrm{~mm}$ and $R=0.3 \mathrm{~mm}$ ) and normal stress $(\sigma=500 \mathrm{kPa})$, as shown in Figures 4 and 5 .

\subsubsection{Influence of temperature on peak adfreezing strength}

As shown in Figures 4 and 5, shear stress increased rapidly at the beginning of the shear progress, and then reached peak shear stress at the relative shear

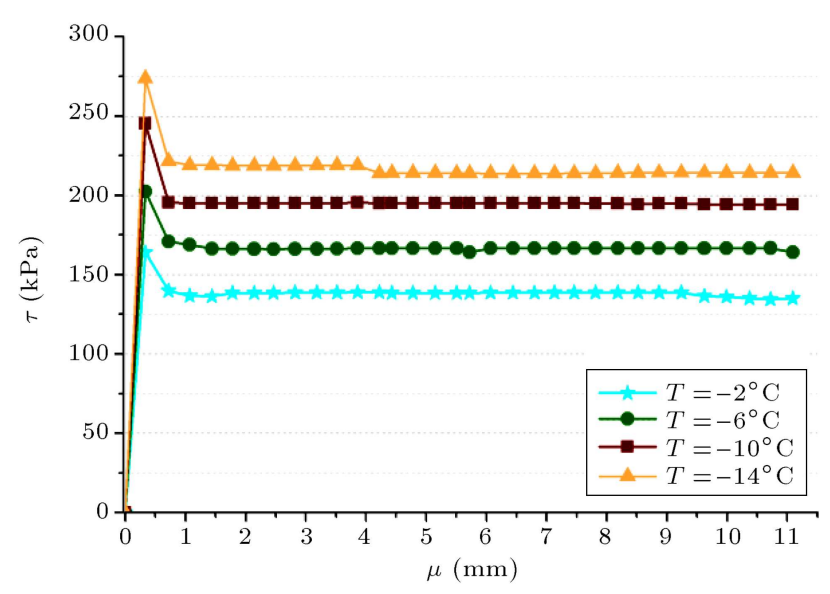

Figure 4. $\tau$ versus $\mu$ curves for each temperature under constant roughness and normal stress $(R=0 \mathrm{~mm}$ and $\sigma=500 \mathrm{kPa})$.

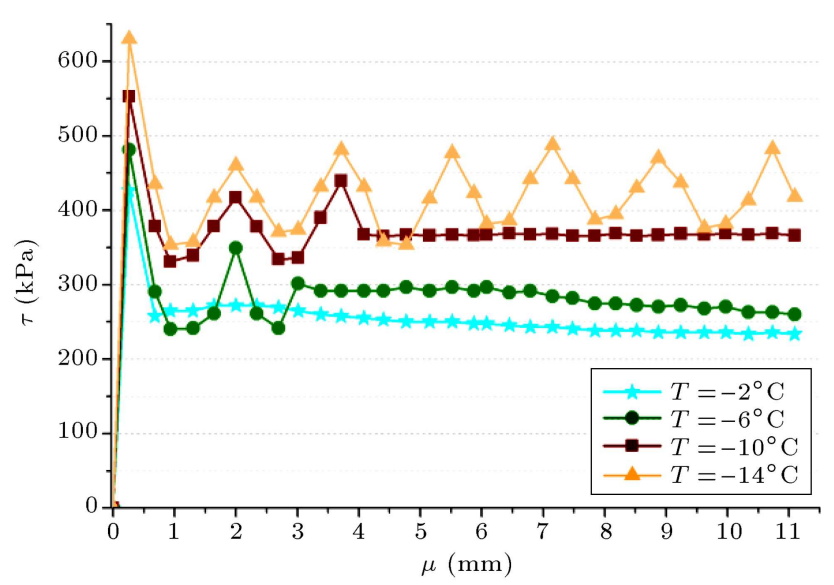

Figure 5. $\tau$ versus $\mu$ curves for each temperature under constant roughness and normal stress $(R=0.3 \mathrm{~mm}$ and $\sigma=500 \mathrm{kPa})$.

displacement of $0.3 \mathrm{~mm}$. This peak shear stress was the peak adfreezing strength as defined earlier. The peak adfreezing strength increased with the decrease of the interface temperature and the increasing rate also increased with it, because the curves corresponding to the increase of the shear stress from zero to the maximum became steeper as the interface temperature decreased. The reason is that the quantity and strength of ice crystals at the interface increased with the decrease of interface temperature, which increases the cementation between ice crystals and shear plate, and also the peak adfreezing strength enhanced at the moment of shear failure.

Figure 6 shows relation lines of peak adfreezing strength with temperature for each normal stress under constant roughness $(R=0.3 \mathrm{~mm})$. This figure shows that the peak adfreezing strength decreased linearly with the increasing interface temperature. The decreasing rate of the peak adfreezing strength along with an increasing interface temperature was closely related 


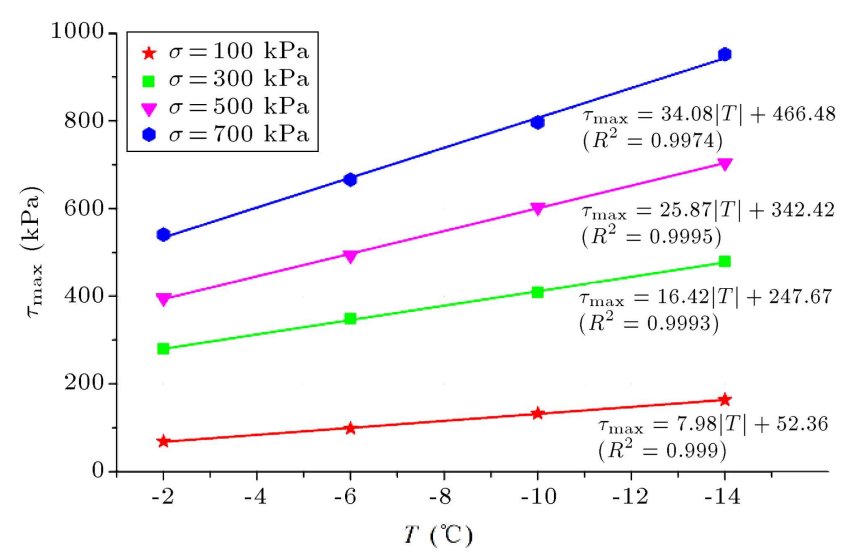

Figure 6. Relation curves of peak adfreezing strength with temperature for each normal stress under constant roughness $(R=0.3 \mathrm{~mm})$.

to normal stress; additionally, the rate increased with increasing normal stress.

The linear relationship between peak adfreezing strength, $\tau_{\max }$, and interface temperature, $T$, could be expressed by Eq. (1):

$$
\tau_{\max }=a \times(-T)+b,
$$

where $a$ and $b$ are constants representing the slope and vertical intercept of fitted curves, respectively. The specific values of $a$ and $b$ are shown in Figure 6 .

\subsubsection{Influence of temperature on residual adfreezing strength}

When the shear plate roughness was $R=0 \mathrm{~mm}$, regardless of interface temperature, the post-peak shear stress, i.e. the residual adfreezing strength, was stable at a relatively constant value with the increase of shear displacement, as shown in Figure 4. The aforementioned issue was brought about when the interface roughness was 0 , the surface of the shear plate was smooth, and the frozen soil at the interface slid smoothly along the surface of the shear plate like sliding friction; therefore, the residual strength was stable at the constant value.

As shown in Figure 5, when the shear plate roughness was $R=0.3 \mathrm{~mm}$, three laws are derived based on the residual adfreezing strength curves. First, when the interface temperature was higher $\left(-2^{\circ} \mathrm{C}\right)$, the frozen soil at the interface was cut off easily in the shearing process due to its lower strength. In this situation, the residual adfreezing strength was just the shear strength of frozen soil; therefore, the residual adfreezing strength curve was stable at a relatively constant value. Second, when the interface temperature was lower $\left(-14^{\circ} \mathrm{C}\right)$, the frozen soil at the interface was not cut off, but slid in fluctuations along the grooves of the shear plate due to its higher strength. Therefore, the residual adfreezing curves showed fluctuating cycles. Third, when the interface temperature was between the above two cases $\left(-6^{\circ} \mathrm{C}\right.$ to $\left.-10^{\circ} \mathrm{C}\right)$, the frozen soil at the interface slid in fluctuations along the grooves of the shear plate at the beginning of the shear. Then, the contact area between the frozen soil and shear plate grooves increased with the increase of sliding distance, and its frictional resistance increased accordingly. Once the friction resistance increased to greater than the shear strength of the frozen soil, the frozen soil at the interface was cut off immediately. Therefore, the residual adfreezing strength first showed a fluctuating variation, and then became stable at a relative constant value.

\subsection{Influence of normal stress on adfreezing strength}

4.2.1. Influence of normal stress on peak adfreezing strength

Figures 7 and 8 show $\tau$ versus $\mu$ curves for each normal stress under constant roughnesses $(R=0.0$ $\mathrm{mm}$ and $R=0.3 \mathrm{~mm}$ ) and constant temperature

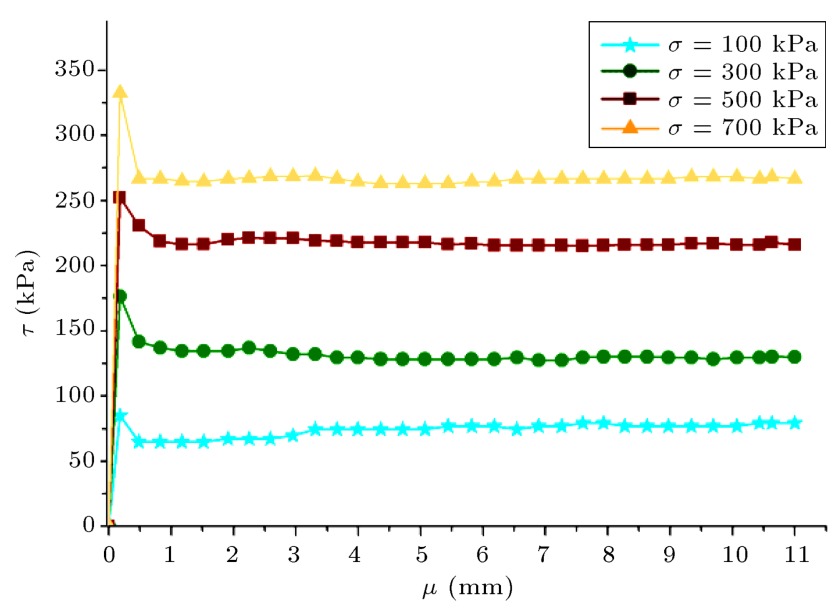

Figure 7. $\tau$ versus $\mu$ curves for each normal stress under constant roughness and temperature $(R=0.0 \mathrm{~mm}$ and $\left.T=-6^{\circ} \mathrm{C}\right)$.

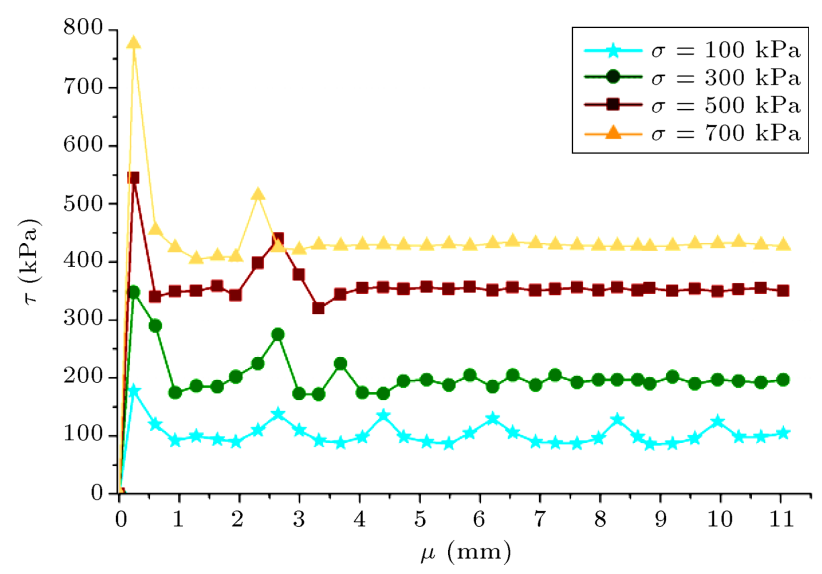

Figure 8. $\tau$ versus $\mu$ curves for each normal stress under constant roughness and temperature $(R=0.3 \mathrm{~mm}$ and $\left.T=-6^{\circ} \mathrm{C}\right)$. 
$\left(T=-6^{\circ} \mathrm{C}\right)$. As shown in those figures, the shear stress increased rapidly after the shear began, and then the peak shear stress (peak adfreezing strength) occurred quickly; all the peak adfreezing strengths increased with the increasing normal stresses. The reasons for the above phenomena were as follows. The frozen soil at the interface was in close contact with the shear plate at the beginning of the shear. Then, the shear stress increased rapidly with the increasing relative shear displacement. When the shear stress further increased to greater than the peak adfreezing strength, relative displacement occurred at the interface between the frozen soil and the shear plate, the bonding force of ice crystals disappeared, the external adfreezing cohesion decreased rapidly, and the static friction was changed into sliding friction; therefore, the post-peak shear stress decreased rapidly. Because the static and sliding friction forces increased with normal stress, although the variation tendencies of ultimate and residual adfreezing strengths were different, they all increased with the increase of normal stress.

Using data of Figures 7 and 8, normalized shear stress curves $(\tau / \sigma$ versus $\mu)$ for each normal stress could be obtained under two kinds of roughness $(T=$ $-6^{\circ} \mathrm{C}$ ), similar to those of Figure 9. This figure shows that the value of $\tau / \sigma$ decreased with the increase of normal stress under two kinds of roughness. This indicated that the contribution of the normal stress to the shear stress reduced as the normal stress increased. Therefore, it was not advisable to increase the shear stress by unduly increasing the normal stress.

Figure 10 shows relation curves of peak adfreezing strength with normal stress for each roughness under constant temperature $\left(T=-10^{\circ} \mathrm{C}\right)$. As shown in the figure, the peak adfreezing strength increased linearly with the increase of normal stress, and its change rate increased with the increase in the roughness value.

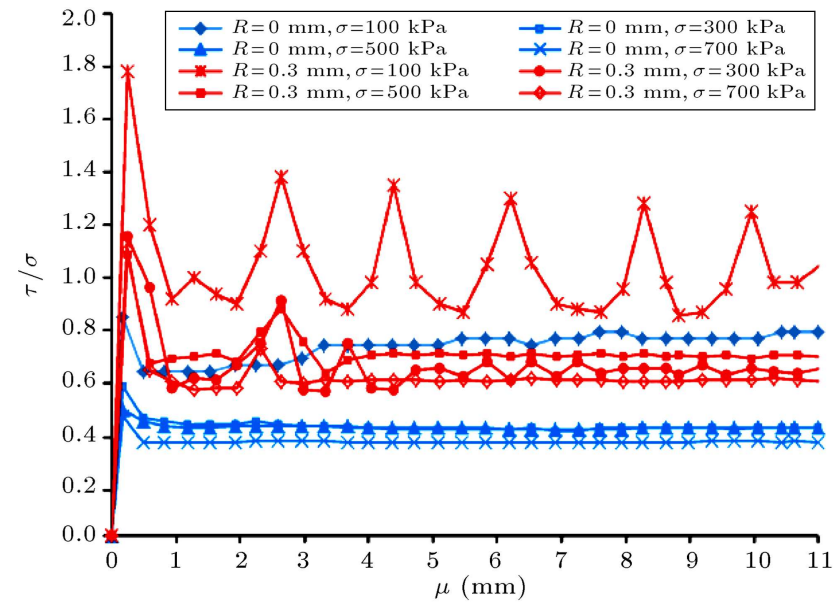

Figure 9. Normalized shear stress curves $(\tau / \sigma$ versus $\mu)$ for each normal stress under two kinds of roughness $\left(T=-6^{\circ} \mathrm{C}\right)$.

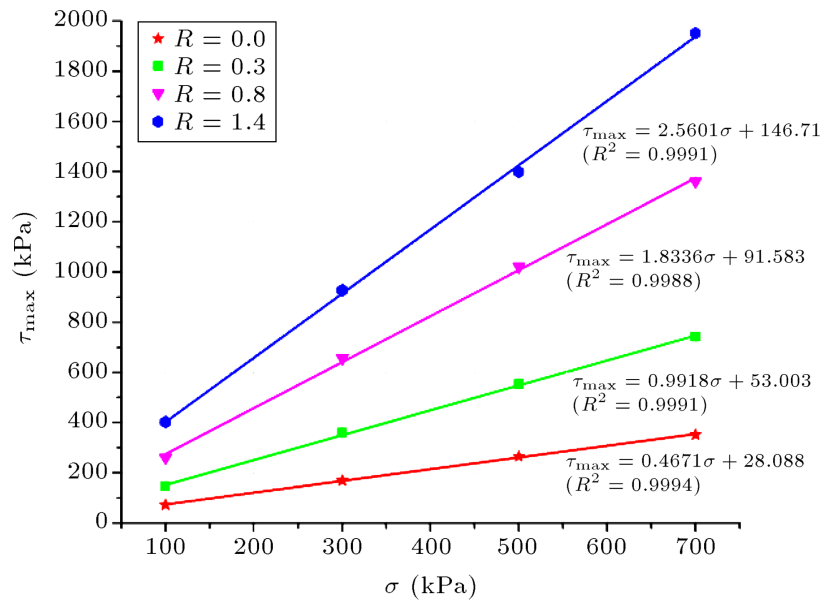

Figure 10. Relation curves of peak adfreezing strength with normal stress for each roughness under constant temperature $\left(T=-10^{\circ} \mathrm{C}\right)$.

As expressed in Eq. (2), peak adfreezing strength, $\tau_{\max }$, and normal stress, $\sigma$, formed a linear relationship, and their relationship was consistent with the MohrCoulomb criterion:

$$
\tau_{\max }=\tan \varphi^{\prime} \times \sigma+c,^{\prime}
$$

where $\varphi^{\prime}$ is external adfreezing friction angle and $c^{\prime}$ is external adfreezing cohesion. In addition, $\varphi^{\prime}$ and $c^{\prime}$ all increased with the increase in roughness value. Herein, $\varphi^{\prime}$ and $c^{\prime}$ are different from the friction angle and cohesion of the soil, and their values are related to the type of soil, contact surface temperature, contact surface roughness, etc.

\subsubsection{Influence of normal stress on residual adfreezing strength}

As shown in Figure 7, when the roughness value was $R=0.0 \mathrm{~mm}$, shear stress rapidly decreased with the increasing shear displacement after the shear stress reached the peak adfreezing strength. When the shear stress decreased by $20 \mathrm{kPa}$ to $65 \mathrm{kPa}$, it was stable at a relatively constant value, which was the residual adfreezing strength. Residual adfreezing strength increased with the increase in normal stress, but it was stable under the same normal stress condition. As shown in Figure 8, when the roughness value was $R=0.3 \mathrm{~mm}$, shear stress also rapidly decreased after the peak shear stress. The decreased amplitude of shear stress was $90 \mathrm{kPa}$ to $350 \mathrm{kPa}$, which was a substantial increase compared to that of a smooth shear plate.

The reasons for the above phenomena are as follows. The greater the roughness of the shear plate, the greater the interface area between the shear plate and the frozen soil. Correspondingly, the adhesion of ice crystals to the interface, the cohesion between the frozen soil and the interface, and the maximum static friction between the frozen soil and the interface all increased, which all led to a significant increase in 
the peak adfreezing strength when the shear plate was rough compared to when it was smooth. The residual adfreezing strength was related to only the sliding friction and the cohesion, but unrelated to the adhesion of ice crystals. Therefore, the increased amplitude of the residual adfreezing strength was smaller compared to the peak adfreezing strength. The different increasing extent between the two kinds of adfreezing strength led to a wider gap between peak shear stress and post-peak shear stress when the shear plate was rough.

The post-peak shear stress suggests two kinds of variation laws. One is a continuous fluctuating cycle and the other is stabilization after the first wave. The reasons are as follows. Under low normal stress, due to the groove lines of the rough shear plate embedded in the frozen soil, the frozen soil was in close contact with the shear plate. During the shearing process, the frozen soil moved along the equally spaced grooves of the shear plate, so the residual adfreezing strength was in a continuous fluctuating cycle. Under high normal stress, the frozen soil still moved along the grooves of the shear plate at the beginning of the shear process. However, due to the higher normal stress, the significant effects of various factors, such as pressure melting of ice, ice melting caused by friction heat, and damage to the soil structure, led to a sharp decrease in the strength of the frozen soil at the interface. Then, the frozen soil at the interface was cut off, and thus the adfreezing strength of the frozen soil at the interface evolved into frozen soil shear strength. Therefore, the value of the residual adfreezing strength remained constant under the same temperature condition. As a result, the normal pattern of the residual adfreezing strength was the varying fluctuations at first, and then a tendency to stabilize.

\subsection{Influence of roughness on adfreezing strength}

\subsubsection{Influence of roughness on peak adfreezing} strength

Figure 11 shows $\tau$ versus $\mu$ curves for each roughness under constant normal stress $(\sigma=300 \mathrm{kPa})$ and constant temperature $\left(T=-10^{\circ} \mathrm{C}\right)$. As shown in Figure 11, the influence of roughness on the adfreezing strength of the frozen soil at the interface was slightly different from that on the two foregoing factors. When the shear process began, shear stress increased rapidly, and soon reached the peak shear stress, which was the peak adfreezing strength. The peak adfreezing strength increased with the increasing roughness. The rate of increase was rapid at first, and then slow and eventually stable, as shown in Figure 12.

By curve fitting, peak adfreezing strength, $\tau_{\text {max }}$, and roughness, $R$, satisfied a logarithmic function, which could be expressed as in Eq. (3):

$$
\tau_{\max }=a \times \ln R+b,
$$

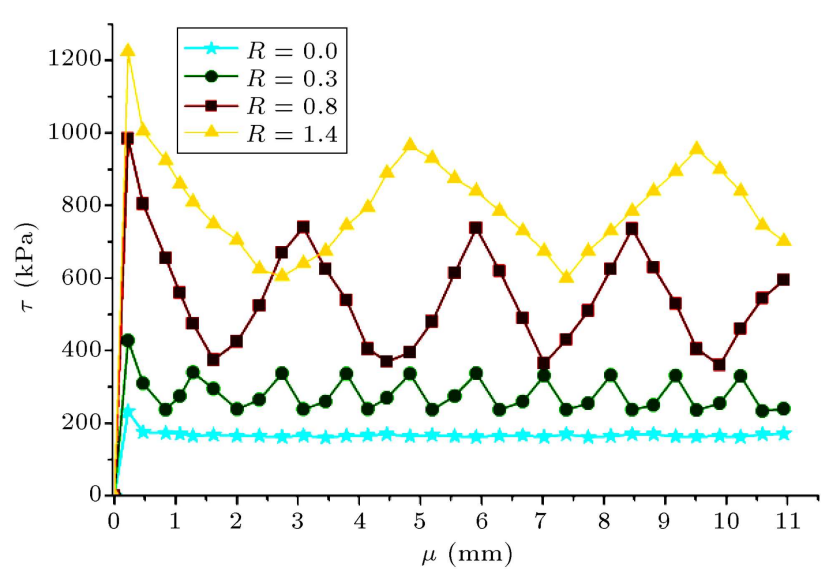

Figure 11. $\tau$ versus $\mu$ curves for each roughness under constant normal stress and temperature $(\sigma=300 \mathrm{kPa}$ and $\left.T=-10^{\circ} \mathrm{C}\right)$.

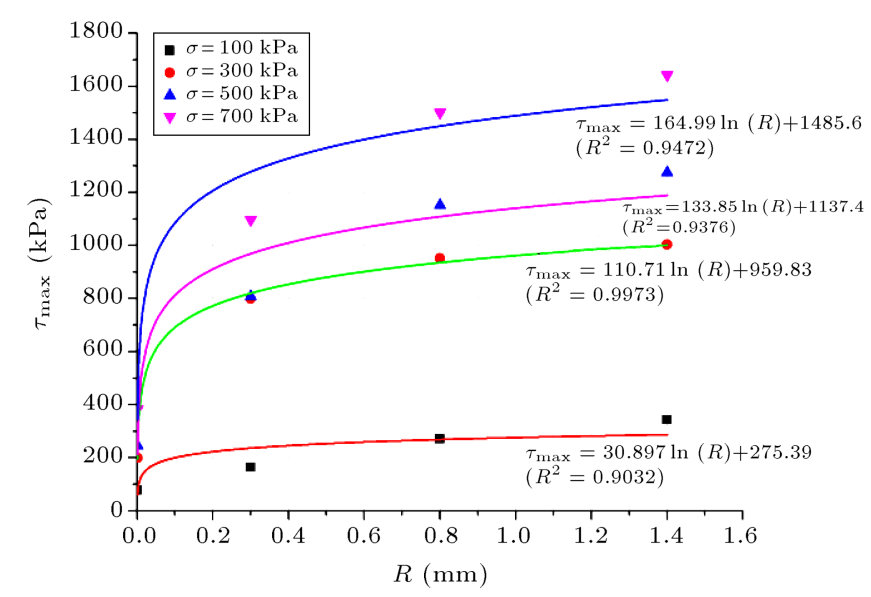

Figure 12. Relation curves of peak adfreezing strength with roughness for each normal stress under constant temperature $\left(T=-10^{\circ} \mathrm{C}\right)$.

where $a$ and $b$ are constant.

The reasons for the above phenomena are as follows. The greater the roughness of the shear plate, the greater the interface area between the shear plate and the frozen soil. Correspondingly, the adhesion of ice crystals to the interface, the cohesion between the frozen soil and the interface, and the maximum static friction between the frozen soil and the interface all increased. When the roughness value continued to increase, the depth of the frozen soil embedded in the grooves of the shear plate also continued to increase; therefore, no relative shear displacement occurred between the frozen soil and shear plate. Therefore, the adfreezing strength evolved into frozen soil shear strength, which was dependent on only interface temperature and normal stress, and unrelated to the value of roughness. Accordingly, the adfreezing strength increased with increasing roughness. The rate of increase was rapid at first, and then slow and eventually stable. 


\subsubsection{Influence of roughness on residual adfreezing strength}

As shown in Figure 11, when the roughness was $R=0$, the residual adfreezing strength stabilized to a constant value. When the roughness was $R>0$, the residual adfreezing strength increased with the increase of roughness and was in a fluctuating cycle; in addition, the length of its cycle increased with the increase of roughness. The reason is as follows. When the roughness was $R=0$, due to the smooth shear plate, there was no normal relative displacement between the frozen soil and the shear plate during the shear process, which was similar to the sliding friction; therefore, the residual adfreezing strength was always stable at a constant value. When the roughness was $R>0$, due to the different deep grooves of the shear plate, there was normal relative displacement between the frozen soil and the shear plate during the shear process, which caused the variation of the residual freezing strength with the relative shear displacement to be in a fluctuating cycle. Because of the different roughness conditions, the spacing of the grooves of the shear plate was different, and the intervals where the frozen soil slipped along the grooves of the shear plate were different. This resulted in different cycle lengths of the residual adfreezing strength, which increased with the increase in roughness. When the roughness value increased, the groove depth of the shear plate increased, and the shear resistance increased accordingly. As a result, the value of residual adfreezing strength increased.

To further explore the relationship between the roughness value of the shear plate and the fluctuating cycle of the residual adfreezing strength, a $\tau$ versus $\mu$ curve $\left(R=0.8 \mathrm{~mm}\right.$ and $\left.T=-10^{\circ} \mathrm{C}\right)$ was arbitrarily selected as the research object, as shown in Figure 13. Average distance, $\bar{L}$, between the adjacent peaks of the residual adfreezing strength curve was $2.69 \mathrm{~mm}(\bar{L}=$ $\left.\left.\left(L_{1}+L_{2}\right) / 2=(2.72+2.65) / 2\right)=2.69 \mathrm{~mm}\right)$.

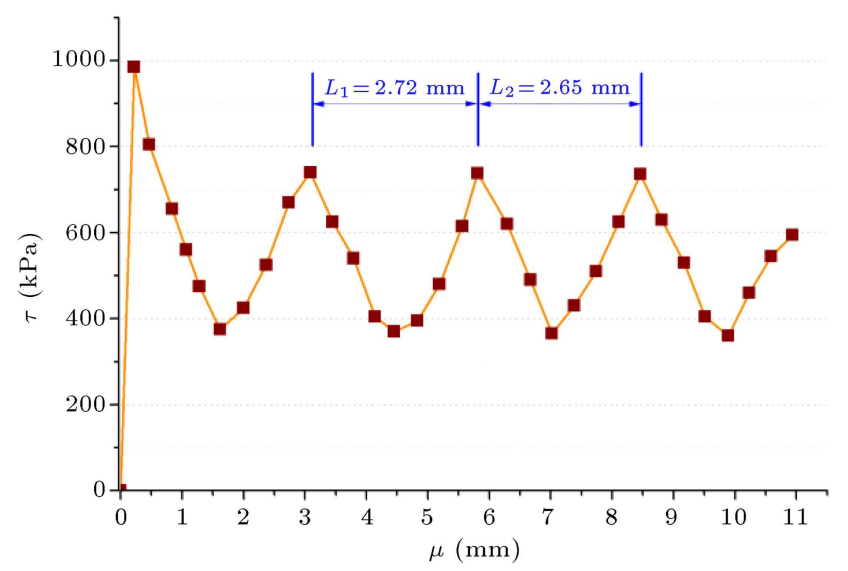

Figure 13. $\tau$ versus $\mu$ curve $\left(\sigma=300 \mathrm{kPa}, T=-10^{\circ} \mathrm{C}\right.$ and $R=0.8 \mathrm{~mm}$ ).

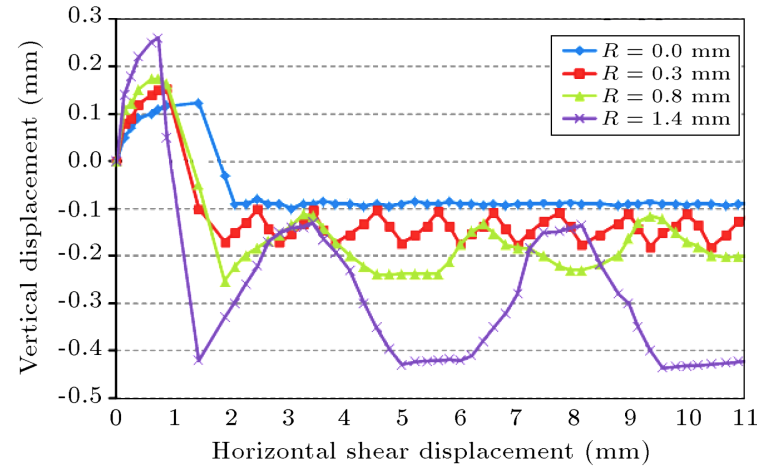

Figure 14. Vertical displacement against horizontal shear displacement $\left(\sigma=300 \mathrm{kPa}\right.$ and $\left.T=-10^{\circ} \mathrm{C}\right)$.

According to the schematic diagram of the rough shear plate (Figure 3) and the definition of the roughness value, distance $L^{\prime}$ between the adjacent teeth of the rough shear plate was $2.73 \mathrm{~mm}\left(L^{\prime}=\sqrt{R}+\right.$ $2 R=1.414 \times 0.8+2 \times 0.8=2.73 \mathrm{~mm})$. Calculation results showed that $L^{\prime}$ and $\bar{L}$ were approximately equal, verifying that the fluctuating cycle of the residual adfreezing strength was due to the fluctuating sliding of the frozen soil at the interface along the grooves of the shear plate.

As shown in Figure 14, the trend of vertical displacement was similar to that of shear stress. As the rough shear plate slid along the dented surface of the frozen soil, the vertical displacement increased as the horizontal shear displacement increased, and the vertical displacement showed cyclical fluctuations when shear plate is rough; moreover, its peak spacing with the shear plate roughness decreased.

The real interface between frozen soil and a structural surface will be quite random and irregular, compared with the grooves used in this study. At present, there are many kinds of methods to calculate the surface grooves of the structure. In most cases, they are calculated by taking the average depth of the grooves in a certain area. It can be understood that the structural grooves are treated as regular and equidistant spacing in the evaluation process. Although the law of residual strength fluctuation in this study is not exactly the same as the actual one, but its general rule is instructive and meaningful in practice.

\section{Formation mechanisms of adfreezing strength}

Based on our experimental research and analysis of the relevant factors influencing adfreezing strengths at the interface between frozen sand and structures, the mechanisms of adfreezing strength formation were revealed.

The peak adfreezing strength consisted of three parts. The first was the bond strength between the 
structure and the crystals formed by water in the soil at negative temperatures. The second was the maximum static friction force at the interface at the critical state of shear failure due to the sliding trend between the frozen soil and the shear plate under the action of a horizontal load. The third was a small amount of adhesion at the interface between the frozen soil and the structure due to the adhesion of the soil itself.

Residual adfreezing strength was the peak shear stress after shear slip failure. When the interface was smooth, the residual adfreezing strength value was a constant. When the interface was rough, the relation curves of residual adfreezing strength with shear displacement showed a state of fluctuating cycles. Therefore, a typical stick-slip phenomenon appeared.

The formation mechanisms of residual adfreezing strength are as follows. Residual adfreezing strength mainly consisted of sliding friction and cohesive force at the interface between the frozen soil and the structure. The stick-slip phenomenon was caused by the different depths and different spacing grooves on the surface of the shear plate with different roughnesses, as shown in Figure 15(a). Due to normal pressure, the frozen soil at the interface was squeezed by the shear plate, so its grooves formed dents in the frozen soil (Figure 15(b)). After the shear stress reached the peak value, the shear plate slipped along the frozen, dented soil surface. In shear process 1 (Figure 15(c)), sliding resistance was relatively large, while, in shear process 2 (Figure $15(\mathrm{~d})$ ), sliding resistance was relatively small. Under the actions of changing sliding resistance, the sliding friction force, i.e. the residual adfreezing strength, was in fluctuating cycles.

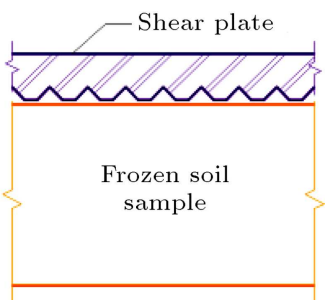

(a) Before normal loading

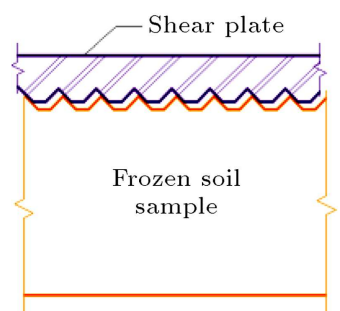

(c) Shear progress 1

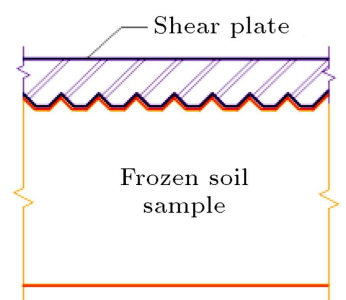

(b) After normal loading

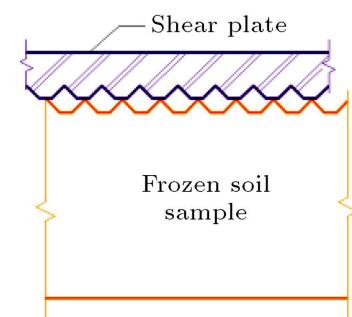

(d) Shear progress 2
Figure 15. Schematic diagram detailing the shearing process.

\section{Construction of empirical formula of peak adfreezing strength}

The analysis of the factors influencing adfreezing strength at the interface was focused on the intrinsic mechanism of adfreezing strength from the perspective of a single factor. However, in actual working conditions, adfreezing strength is affected by multiple factors at the same time; therefore, it was necessary to comprehensively analyze all factors influencing adfreezing strength.

According to the correlation analyses mentioned earlier, the relationship between peak adfreezing strength, $\tau_{\max }$, and surface temperature, $T$, and that between peak adfreezing strength $\tau_{\max }$ and normal stress, $\sigma$, were all linear, as shown in Eqs. (1) and (2). The relationship between peak adfreezing strength, $\tau_{\text {max }}$, and interface roughness, $R$, was logarithmic, as shown in Eq. (3). Therefore, relationships between $\tau_{\max }$ and $T, \sigma$ and $R$ could be assumed as in Eq. (4):

$$
\tau_{\max }=a \times|T|+b \times \sigma+c \times \ln R+d,
$$

where $a, b, c$, and $d$ were all constants to be determined. Parameter $R$ for an interface is generally measured by roughness measurement, and it is calculated by taking the average depth of the grooves on interface in a certain area.

To determine the values of the four constants in Eq. (4), MATLAB software was used to carry out multivariate nonlinear regression analysis. Based on the functional characteristics of Eq. (4), the mathematical model was initially built as shown in Eq. (5):

$$
y=\beta_{1} x_{1}+\beta_{2} x_{2}+\beta_{3} x_{3}+\beta_{4} .
$$

A total of 64 sets of test data on the peak adfreezing strength were sampled. Based on $y$ and $\tau_{\max }$, $x_{1}$ and $T, x_{2}$ and $\sigma$, and $x_{3}$ and $R, 4$ arrays were built first. By calculating the multiple nonlinear regression using the MATLAB nlinfit function, the regression coefficients were calculated: $\beta 1$ was $41.9898, \beta 2$ was $1.2356, \beta 3$ was 97.9437 , and $\beta 4$ was -1.7194 . The value of Rmse (residual standard deviation) obtained by the MATLAB export function was 26.2575. Interactive pictures of calculation results are shown in Figure 16.

The calculation results showed that the ratio between $R$ mse (the value of residual standard deviation, 26.2575 ) and $\bar{\tau}_{\max }$ (the average value of peak adfreezing strength, 632.5938) was $4.15 \%$. The interactive pictures of calculation results show that the linear relationships between $y$ and $x_{1}, x_{2}$, and the logarithm relationships between $y$ and $x_{3}$ were substantial, and their confidence intervals were reasonable. Therefore, the mathematical model (Eq. (5)) drafted preliminarily was certainly reasonable, and the calculation results were valid. Based on the above analyses, the 


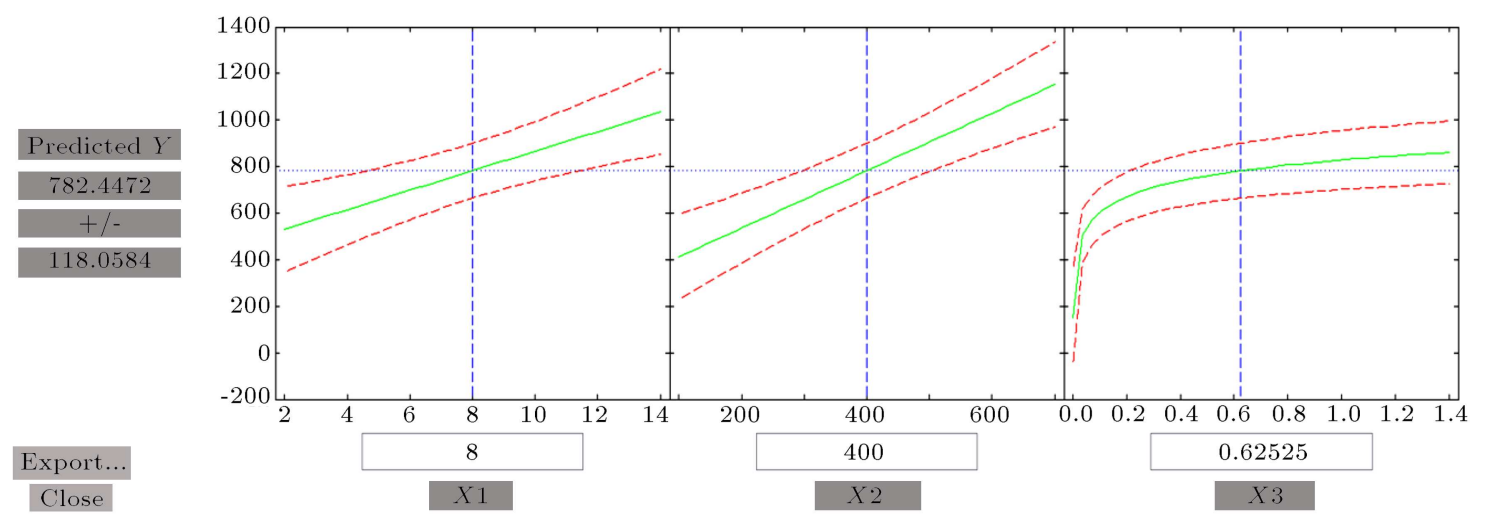

Figure 16. Interactive pictures of calculation results regressed by MATLAB software.

empirical formula of peak adfreezing strength, $\tau_{\max }$, at surface temperature, $T$, normal stress, $\sigma$, and interface roughness, $R$, is as shown in Eq. (6):

$$
\tau_{\max }=41.99 \times|T|+1.24 \times \sigma+97.94 \times \ln R-1.72 .
$$

Therefore, under any condition of known values of interface temperature, normal stress, and roughness, the value of peak adfreezing strength could be predicted by Eq. (6).

\section{Conclusions}

The main research conclusions are as follows:

- The adfreezing strengths at the interface between frozen sand and structures were defined as peak adfreezing strength and residual adfreezing strength;

- The relations between peak adfreezing strength, $\tau_{\max }$, and interface temperature, $T$, satisfied positive linear functions. The variations of residual adfreezing strength with interface temperature presented three typical regular patterns: sustained stable, first stable and then fluctuating, and circularly fluctuating;

- The relationship between peak adfreezing strength, $\tau_{\max }$, and normal stress, $\sigma$, was in line with the Mohr-Coulomb criterion. The internal friction angle and cohesive strength increased with the increase in roughness;

- Peak adfreezing strength and residual adfreezing strength both increased with an increase in roughness value. The relations between peak adfreezing strength, $\tau_{\max }$, and roughness, $R$, satisfied logarithmic functions;

- Adfreezing strength formation mechanisms were revealed. Residual adfreezing strength produced a stick-slip phenomenon due to the grooved shear plate surface slipping along the dented surface of the frozen sand;
- An empirical formula on peak adfreezing strengths was constructed. This formula might be used to predict the value of peak adfreezing strengths and provide a basis for the design of shield segments and the advanced construction of tunnel boring machines.

\section{Acknowledgments}

This work was supported by the National Natural Science Foundation of China (Grant No. 51278251), the Construction Project of Dominant Subjects in Colleges and Universities of Jiangsu Province, and the Research Project of Ministry of Housing and UrbanRural Development of China (2014-k3-029).

\section{References}

1. Lashkari, A. "Prediction of the shaft resistance of nondisplacement piles", International Journal for $\mathrm{Nu}$ merical and Analytical Methods in Geomechanics, 37, pp. 904-931 (2013).

2. Kadivar, M. and Lashkari, A. "A constitutive model for unsaturated soil-structure interfaces", International Journal for Numerical and Analytical Methods in Geomechanics, 40, pp. 207-234 (2016).

3. Lashkari, A. "A critical state model for saturated and unsaturated interfaces", Scientia Iranica, 19(5), pp. 1147-1156 (2012).

4. Dejong, J.T. and Westgate, Z.J. "Role of initial state, material properties, and confinement on local and global soil structure behavior", ASCE J. Geotechnical Geoenvironmental Eng.., 135, pp. 1646-1660 (2009).

5. Miller, G.A. and Hamid, T.B. "Direct shear apparatus for unsaturated soil interface testsing", ASTM GTJ, 30, pp. 182-191 (2007).

6. Suits, L.D., Sheahan, T.C., Porcino, D., et al. "Interface behavior of sands from constant normal stiffness direct shear tests", ASTM GTJ, 26, pp. 1-13 (2003). 
7. Dove, J.E. and Jarrett, J.B. "Behavior of dilative sand interfaces in a geotribology framework", $A S C E$ J. Geotechnical Eng., 128, pp. 25-37 (2002).

8. Evgin, E. and Fakharian, K. "Effect of stress path on the behavior of sand-steel interface", Canadian Geotechnical Journal, 33, pp. 853-865 (1996).

9. Yin, Z., Hong, Z., and Xu, G. "A study of deformation in the interface between soil and concrete", Computers and Geotechnics, 17, pp. 75-92 (1995).

10. Parameswaran, V.R. "Adfreeze strength of frozen sand to model piles", Canadian Geotechnical Journal, 15(4), pp. 494-500 (1978).

11. Ladanyi, B. and Theriault, A. "A study of some factors affecting the adfreeze bond if piles in permafrost", Proceedings of Geotechnical Engineering Congress, GSP 27 ASCE, 1.1, pp. 213-224 (1990).

12. Terashima, T., Kawai, T., Furuya, A., et al. "Experimental study on adfreeze bond strength between ice and pile structure", Proceedings of the 9th International Offshore and Polar Engineering Conference, Brest, France, pp. 549-556 (1999).

13. Weaver, J.S. and Morgenstern, N.R. "Pile design in permafrost", Canadian Geotechnical Journal, 18(3), pp. $357-370$ (1981).

14. Zhang, J., Zhu, Y., and Zhang, J. "Adfreeze strength of model piles in frozen soil under dynamic loads", Proceedings of the Seventh Permafrost International Conference, Yellowknife, Canada, pp. 1217-1221 (1998).

15. Liu, J.K., Cui, Y.H., Wang, P.C., et al. "Design and validation of a new dynamic direct shear apparatus for frozen soil", Cold Regions Science and Technology, 106, pp. 207-215 (2014).

16. Lv, P., Liu, J.K., and Cui, Y.H. "A study on dynamic shear strength on frozen soil-concrete interface", Sciences in Cold and Arid Regions, 5(4), pp. 408-412 (2013).

17. Biggar, K.W. and Sego, D.C. "The strength and deformation behaviour of model adfreeze and grouted piles in saline frozen soils", International Journal of Rock Mechanics and Mining Sciences \& Geomechanics Abstracts, 31(1), pp. 319-337 (1994).

18. Ueda, Y.K., Moriuchi, K., and Ohrai, T. "Influence of normal stress on the adfreeze interface on adfreeze shear strength of frozen soil", Journal of the Japanese Society of Snow \& Ice, 66, pp. 197-205 (in Japanese) (2004).

19. Choi, C.H. and Ko, S. "A study for predicting adfreeze bond strength from shear strength of frozen soil", Journal of the Korean Geotechnical Society, 27(10), pp. 13-23 (in Korean) (2011).
20. Lee, J., Kim, Y., and Choi, C. "A study for adfreeze bond strength developed between weathered granite soils and aluminum plate", Journal of the Korean GeoEnvironmental Society, 14(12), pp. 23-30 (2013) (in Korean).

21. Ko, S.G. and Choi, C.H. "Experimental study on adfreeze bond strength between frozen sand and aluminium with varying freezing temperature and vertical confining pressure", Journal of the Korean Geotechnical Society, 27(9), pp. 67-76 (2011) (in Korean).

22. Lee, J.Y. and Choi, C.H. "A study for shear strength characteristics of frozen soils under various temperature conditions and vertical confining pressures", Journal of Korean Geo-Environmental Society, 13(11), pp. 51-60 (2012) (in Korean).

23. Lee, J.Y. and Choi, C.H. "Shear strength characteristics of weathered granite soil below the freezing point", Journal of Korean Geo-Environmental Society, 14(7), pp. 19-29 (2013) (in Korean).

24. Liu, J., Lv, P., Cui, Y. et al. "Experimental study on direct shear behavior of frozen soil-concrete interface", Cold Regions Science and Technology, 104-105, pp. 1-6 (2014).

25. Zhao, L., Yang, P., and Wang, H. "Development and application of large-scale multi-functional frozen soilstructure interface cycle-shearing system", Chinese Journal of Geotechnical Engineering,(04), pp. 707-713 (in Chinese) (2013).

26. Zhao, L., Yang, P., Wang, J., et al. "Cyclic direct shear behaviors of frozen soil-structure interface under constant normal stiffness condition", Cold Regions Science and Technology, 102, pp. 52-62 (2014).

27. Zhao, L., Yang, P., Wang, J., et al. "Impacts of surface roughness and loading conditions on cyclic direct shear behaviors of an artificial frozen silt-structure interface", Cold Regions Science and Technology, 106107, pp. 183-193 (2014).

28. Sun, H., Yang, P. and Wang, G. "Development of mechanical experimental system for interface layer between frozen soil and structure and its application", Rock and Soil Mechanics, 35(12), pp. 3636-3641+3643 (2014) (in Chinese).

29. Qiu, G., Liu, J., Liu, H. "Geocryological glossary", Gansu Science and Technology Press, Lanzhou, pp. 115-117 (1994) (in Chinese).

\section{Biographies}

Shi Quanbin received his BSc degree in Traffic Civil Engineering from Hefei University of Technology, Hefei, China in 2000, and obtained his MSc degree in Civil Engineering from Nanjing Forestry University, Nanjing, China in 2012. He is currently studying for a $\mathrm{PhD}$ at Nanjing Forestry University. He is an Associate Professor in Civil Engineering Department at Taizhou Polytechnic College. He has 16 years of teaching experience in Soil Mechanics, Foundation Engineering 
and Traffic Civil Engineering at both undergraduate and graduate courses. He has authored and coauthored more than a dozen research papers. His main research areas are foundation engineering, artificial frozen soil theory, and tunnel construction.

Yang Ping received his BSc degree in Civil Engineering from Anhui University of Science and Technology, Anhui, China in 1985, MSc degree in Civil Engineering from Anhui University of Science and Technology, Anhui, China in 1988. He is currently a Professor in Civil Engineering Department at Nanjing Forestry University. He has published 2 monographs on artificial frozen soil and tunnel construction. He has won scientific and technological progress award 7 times at the provincial and ministerial level. He has 28 years of teaching experience in Soil Mechanics, Foundation Engineering, Environmental Geotechnical Engineering, and Urban
Underground Engineering at both undergraduate and graduate courses. He has authored and coauthored more than 140 research papers in various journals and international conferences. His main research areas are environmental geotechnical, soil mechanics, artificial frozen soil theory and artificial freezing technology.

Wang Guoliang received his BSc in Civil Engineering from Nanjing Forestry University, Nanjing, China, in 2013, and obtained his MSc degree in Geotechnical Engineering from Nanjing Forestry University, Nanjing, China in 2015. He is currently working in Xuzhou metro construction management department. He carried out his research thesis titled "The research on the frozen strength of the artificial frozen silt-structure interface" under supervision of Professor Yang Ping. His research achievements have been quite significant, and he has already published four research papers. 